\title{
Naegleria: a classic model for de novo basal body assembly
}

Lillian K. Fritz-Laylin ${ }^{1 *}$ (D) and Chandler Fulton ${ }^{2}$

\begin{abstract}
The amoeboflagellate Naegleria was one of the first organisms in which de novo basal body/centriole assembly was documented. When in its flagellate form, this single-celled protist has two flagella that are templated by two basal bodies. Each of these basal bodies is structurally well conserved, with triplet microtubules and well-defined proximal cartwheel structures, similar to most other eukaryotic centrioles. The basal bodies are anchored to the nucleus by a single, long striated rootlet. The Naegleria genome encodes many conserved basal body genes whose expression is induced prior to basal body assembly. Because of the rapid and synchronous differentiation from centriole-less amoebae to temporary flagellates with basal bodies, Naegleria offers one of the most promising systems to study de novo basal body assembly, as well as the mechanisms regulating the number of centrioles assembled per cell.
\end{abstract}

\section{The organism}

Naegleria gruberi is a free-living protist easily isolated from freshwater sources around the world [1-3]. Naegleria's reproductive form is a $15-\mu \mathrm{m}$ predatory amoeba that feeds on bacteria (Fig. 1). However, when faced with environmental signals such as nutritional, temperature, osmotic, and/or pH shifts, Naegleria undergoes an astounding metamorphosis from a crawling amoeba to a streamlined flagellate capable of swimming for several hours before reverting to an amoeba [2,3]. Only the amoebae reproduce, and their mitosis involves no centrioles [4]. The amoeba-to-flagellate differentiation requires de novo assembly of basal bodies and flagella, including transcription and translation of their molecular components, even including tubulin (Fig. 1) [5-9]. Despite the complexity of this task, Naegleria cells accomplish the amoeba-to-flagellate conversion in about an hour [2, 3]. This developmental feat led to one of the first discoveries of de novo basal body assembly [4], at a time when even the concept of de novo centriole assembly was met with scepticism. To this day, one of the most interesting

\footnotetext{
*Correspondence: fritzlaylin@gmail.com

${ }^{1}$ Department of Cellular and Molecular Pharmacology, University of California, San Francisco, CA 94158, USA

Full list of author information is available at the end of the article
}

features of Naegleria centrioles is the speed at which differentiating cells turn on the genes, synthesize the proteins, and assemble two canonical basal bodies without any pre-existing "template" precursors. Naegleria synthesizes and assembles centriole components only during the transition to its temporary flagellate form; in the laboratory, at least, it can live for years as reproducing amoebae or resting cysts without ever using centrioles.

Naegleria has been developed as a model to study its incredibly rapid, synchronous, and reproducible differentiation from one cell phenotype to a very different one. Protocols have been developed for straightforward control of this process [2, 3], a methodology that opened the door to understanding the roles transcription and translation play in de novo centriole assembly [10], and tracing the expression, translation, and localization of individual proteins during differentiation [5-8]. More recently, genome sequencing has revealed that Naegleria has many canonical centriole/basal body genes, and microarray analysis of differentiation has also led to the prediction of novel centriole genes $[9,11]$.

Naegleria is a member of the heteroloboseans, a clade composed of a wide variety of amoebae, flagellates, and amoeboflagellates, of which Naegleria is the best-studied example [11]. The heteroloboseans are distantly related to two other groups, the jacobids, and the euglenozoans 


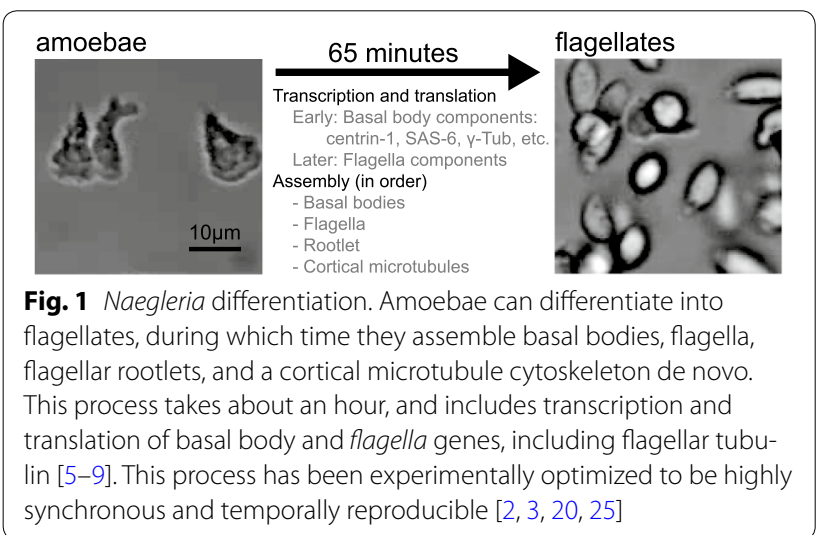

that include the parasitic trypanosomes [12]. The ancestor of these three clades diverged from other eukaryotic lineages somewhere during the past $1-3$ billion years $[11$, 13].

Despite the eons that separate Naegleria from animal and fungal lineages, analysis of its fully sequenced genome indicates that Naegleria represents a sophisticated and surprisingly complex modern eukaryote, with about 16,000 genes including complete actin and microtubule cytoskeletons, mitotic and meiotic machinery, transcription factors [14], membrane trafficking, extensive networks of signaling machinery (including hundreds of protein kinases and small GTPases), and both aerobic and anaerobic metabolic pathways [11].

The genus Naegleria has about 40 species that are defined mainly by differences in extrachromosomal DNA sequences [15]. Many of these have very similar life histories, although there are some less-studied species that appear to have other options in their life cycles (such as division in flagellates [1]). Clonal strains of two morphologically very similar free-living species have been used for almost all studies of basal body development and form. One is N. gruberi strain NEG (the strain for which we have a draft genome [11]); the other was also known as $N$. gruberi strain NB-1 until a difference in ITS sequence caused it to be redefined as $N$. pringsheimi [15]. Herein when we refer to Naegleria we are referring to studies in strains NEG and NB-1. (The opportunistic human pathogen $N$. fowleri has a similar life cycle, and when it forms flagellates the basal bodies appear to be formed de novo $[16,17])$.

\section{Basic basal body structure}

Mature Naegleria flagellates typically have two basal bodies that are anchored at the plasma membrane and template motile flagella [18]. The two basal bodies appear structurally equivalent, with triplet microtubules and a clear luminal cartwheel at the proximal end (Fig. 2) [18].

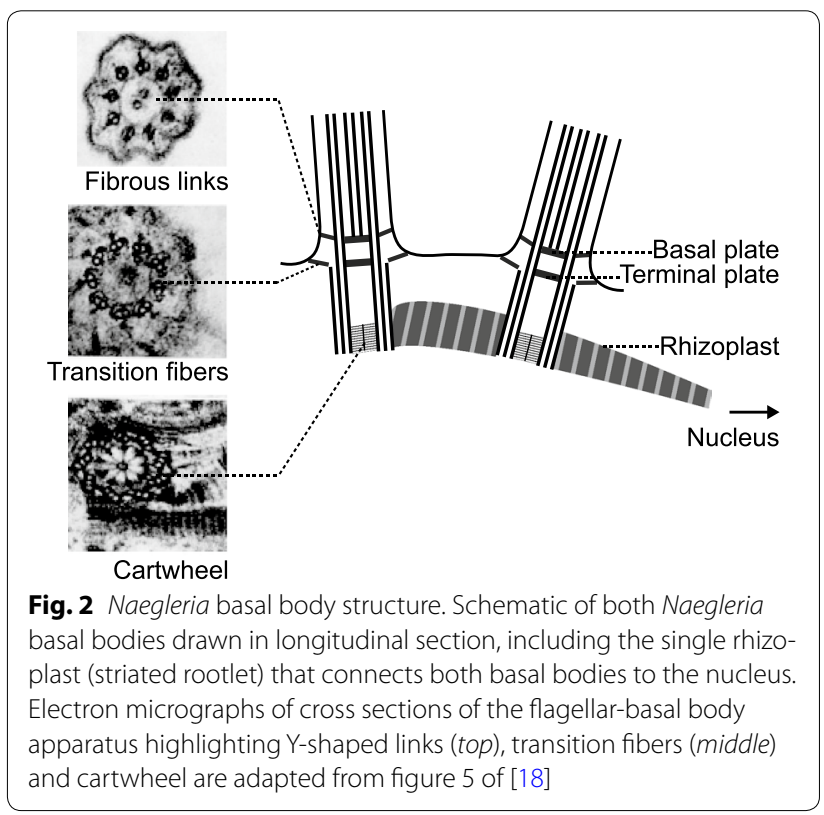

Consistent with this canonical centriole ultrastructure, the Naegleria genome encodes many conserved centriole components, including $\gamma^{-}, \delta$-, and $\varepsilon$-tubulins, and SAS- 6 [11]. These and other core components are readily recognized, although some Naegleria orthologs have extensively diverged from those of commonly studied species.

Based on a seminal electron microscopy study of Naegleria basal bodies and flagella [18], transition zones also appear well conserved. Although electron micrographs revealing details of the lumen of the transition zone are not available, the published data clearly show electron densities representing both basal and terminal plates [18]. Fibrous links between microtubule doublets and the membrane can be seen at the level of the basal plate, likely corresponding to the Y-shaped links seen at this location in other organisms, connecting microtubule doublets to the ciliary neck. Proximal to the terminal plate, fibers radiate from microtubule triplets into the cytoplasm, which are likely transition fibers [18].

\section{Additional basal body structures or accessory structures}

Naegleria's dual basal bodies are connected to its nucleus by a slender, long (up to 15 microns) striated rootlet called a rhizoplast (Fig. 2) [18-20]. One end of the rhizoplast is tightly adhered to the proximal end of the basal bodies via a striated wedge-shaped structure, while the other end runs along the nucleus, terminating in a pocket within the nuclear envelope [18].

The strength of the attachment of the rhizoplast to the basal bodies is evident by the ability of the two to be 
purified intact [19, 21]. Even the complex of nucleus and flagellar apparatus (basal bodies, rootlets, flagella) are sufficiently attached to be co-isolated [18]. Purified rhizoplasts appear to be at least $50 \%$ composed of a single $170 \mathrm{KD}$ protein, and have been suggested to be related to striated ciliary rootlets of other organisms $[19,21]$. The major rootlet protein is synthesized de novo during differentiation, and the rootlet is assembled 5-6 $\mathrm{min}$ after the flagella become visible [20].

\section{Basal body origins}

In Naegleria, basal bodies are transient structures, assembled during the amoeba-to-flagellate differentiation, functional for several minutes to hours, and then disassembled during the de-differentiation to the amoeboid form [2, 3, 8]. Electron microscopy studies of synchronously differentiating cells indicate that both basal bodies are built within minutes, about $10 \mathrm{~min}$ before flagella emerge [4]. This rapid de novo basal body assembly has been of interest for some time, and there are a number of studies focused on understanding the required molecular events.

Studies of Naegleria orthologs of known basal body proteins (Northern blots to measure their mRNAs, and Western blots and immunoflorescence using affinitypurified polyclonal antibodies raised to Naegleria proteins) along with other experiments, including chemical inhibition of translation, have shown that Naegleria basal body assembly occurs by stepwise assembly of conserved components that are transcribed and translated de novo $[3,4,6-9,22]$. In several cases, it is clear that a cluster of basal body genes are coexpressed earlier in differentiation than the cluster of genes required for flagellar assembly, events defined in detail for centrin-1, SAS6 , and $\gamma$-tubulin $[7,8]$. Together, these studies indicate that Naegleria basal body assembly proceeds in roughly the same order of events as during centriole assembly in Chlamydomonas or human cells. This conclusion is supported by full genome transcriptional profiling showing robust and rapid induction of known centriole genes during differentiation [9].

\section{Basal body life cycle and other functions}

Naegleria basal bodies, like the rest of its cytoplasmic microtubule cytoskeleton, are assembled during the transition to the flagellate form and disassembled upon transition back to an amoeba $[2,3,20]$. Naegleria undergoes mitosis and cytokinesis as an amoeba, where there are no centrioles or basal bodies present [4, 23, 24]. It therefore represents an interesting case of centriole assembly outside of the cell cycle. Because Naegleria routinely reproduces for hundreds of generations in its amoeboid form without ever building or containing a centriole/ basal body $[4,25]$, this organism clearly does not require a basal body or centriole for its normal growth. Mitosis in Naegleria is intranuclear, and the microtubules do not focus to the poles $[4,24,26]$. It is clear that the basal body does not assume the role of a centrosome, and there is no hint that any other structure serves to focus the mitotic microtubules.

However, in addition to templating the flagella [18], the basal bodies do seem to act as microtubule organizing centers in the flagellate, where a focus of $\gamma$-tubulin enrichment has been observed, from which emanates a large "cage" of microtubules which follows the cortex of the cell $[8,27,28]$.

A genus of free-living amoeboflagellates closely related to Naegleria, Tetramitus, shows some striking differences from Naegleria. Like Naegleria, Tetramitus can differentiate from centriole-less amoebae to flagellates, in this case with four basal bodies and four flagella [2, 29]. The differentiation is slower, and requires a microaerobic environment [30]. Most strikingly, the flagellates can also become stable and reproduce, so that this species can assume two stable, reproducing phenotypes: amoebae and flagellates [2]. The ultrastructure of the flagellates has been described, and preliminary observations suggest that division in the flagellates is also acentriolar, and in particular that the basal bodies do not appear to participate in division [31,32]. Surprisingly, this fascinating genus has been little studied to date.

\section{Identification of basal body components}

Centrin has long been known to be associated with Naegleria basal bodies [7,33], which have been more recently shown to contain SAS-6 [8]. Although proteomics of purified Naegleria basal bodies has not been reported, the mass induction of basal body genes during differentiation has been used to predict conserved and novel basal body proteins, including: $\delta$ - and $\varepsilon$-tubulins, SAS-4/CenPJ/CPAP and SAS-6, POC1, POC11, POC12, POC16, MKS1, and MKS3 [9]. Naegleria, like other eukaryotic species with motile flagella, also has conserved Pix proteins [34].

\section{Notable basal body findings}

As has been discussed above, Naegleria was one of the first reported cases of de novo basal body assembly [4], and for decades remained the best-studied example. It was also by studying Naegleria differentiation, in particular the induction of $\alpha$ - and $\beta$-tubulin isoforms specific to flagellates, that led to the origin of the multitubulin hypothesis, which predicted the existence of multiple types of tubulin that would be used to build different cellular structures [5]. Both flagellar $\alpha$ - and $\beta$-tubulins, which are incorporated into basal bodies, flagella, and 
cortical microtubules, undergo highly regulated synthesis during differentiation [3, 5, 22, 35, 36]. Evidence has been presented that another, very divergent, $\alpha$-tubulin is used for mitosis in Naegleria [37].

An area of great promise for future research in Naegleria is how the majority of differentiating Naegleria cells assemble exactly two basal bodies and two flagella. There are already some provocative observations in the literature that hint at an interesting counting mechanism.

Naegleria strain NEG is normally diploid (2n) [11], but in culture it often becomes tetraploid (4n), presumably due to failure of mitotic nuclei to separate [2] (p. 459). While the diploid strains tend to have two flagella (2n2f), the tetraploids initially tend to have four flagella (4n4f). This configuration is metastable, however, and after some growth in culture tetraploid cells tend to revert to forming two flagella upon differentiation (i.e., $4 \mathrm{n}-2 \mathrm{f}$ ). In this state, they look very similar to strain NB-1, which is a stable tetraploid that typically makes two flagella (i.e., $4 n-2 f)$. In both cases, $4 n-2 f$ cells seem to have looser control over their counting, with around $20 \%$ flagellates having 3-4 flagella, compared to only $2 \%$ of 2 n-2f NEG flagellates [2] (p. 413). These simple observations are easily reproduced $[2,25]$, but perhaps more challenging to understand. While ideas of possible precursors that divide along with cell division are appealing [38] (p. 199), they do not seem necessary since known proteins seem sufficient to nucleate the formation of a new basal body independent of any precursor structure (e.g., [39, 40]).

Strikingly, sublethal temperature shocks at appropriate times during differentiation can dramatically increase the number of basal bodies and flagella that Naegleria assembles [41, 42]. For example, on average strain NB-1 normally assembles 2.2 flagella. However, after a $38^{\circ}$ temperature shock, this average rises to 4.5 , with a range of up to 18 flagella on a single cell [41]. These multiflagellate cells display disorganized swimming and tumbling. When these flagellates revert to amoebae in the same nonnutrient environment, they immediately redifferentiate without division, but with only the normal number of flagella (average of 2.1) [41]. Why heat-shock temporarily alters flagellar number, as well as the nature of the normal control mechanism, remain interesting challenges for future investigation.

In three published reports from JooHun Lee's laboratory, it has been suggested that a novel entity regulates Naegleria basal body assembly in an unprecedented manner [43-45]. Their work presents evidence that Naegleria amoebae maintain a novel protein complex through numerous generations. This complex, containing a Naegleria transacetylase protein, is reported to accumulate $\gamma$-tubulin, pericentrin, and myosin II. The resulting "GPM" complex, present in amoebae, moves to the site of basal body assembly, and provides the focus where two basal bodies form de novo. Then the complex (including $\gamma$-tubulin) leaves the site of basal body assembly, travels to the other end of the cell, and disassembles, leaving the basal bodies behind. In this study, the presence of $\gamma$-tubulin is used to build the hypothesis that the complex might transiently nucleate the start of basal body assembly. Although provocative, the reliance on mammalian antibodies without properly defined epitopes in Naegleria to trace the movement and fate of the GPM complex leaves room for serious disagreement with these findings. In the experience of our laboratories, Naegleria proteins are sufficiently divergent from other species that the immunofluorescence signal when using heterologous antibodies (if there is any) is almost always to unknown antigens, or proteins trapped at the posterior end of amoebae (e.g., [8]). Specifically, both our labs have tried heterologous antibodies to $\gamma$-tubulin, without success. This is in stark contrast to results obtained by using affinity-purified antibodies raised to the single Naegleria $\gamma$-tubulin gene product. These antibodies reveal that $\gamma$-tubulin is localized to the basal bodies during their assembly, and remains stably localized there-parallel to the result observed for $\gamma$-tubulin in other species [8]. In addition, our results indicate that $\gamma$-tubulin, like other basal body proteins, is not present in amoebae: the mRNA for $\gamma$-tubulin is induced early in differentiation [9], and $\gamma$-tubulin antigen accumulates as the basal bodies are assembled [8]. The fact that Lee's results show the heterologous antibody epitopes are already present in amoebae, and go on to dissociate from the basal bodies, make it seems likely to us that the recognized epitope is not $\gamma$-tubulin. In their most recent paper [44], Lee et al. used a new antibody to a Naegleria $\gamma$-tubulin peptide, but in immunogold electron microscopy found that this antibody did not colocalize with the structure recognized by the heterologous $\gamma$-tubulin antibody they had used to define the GPM complex. (Similar objections apply to the heterologous pericentrin antibody they used; in this case it is also unknown what epitope is staining, and no pericentrin gene has been curated in the Naegleria genome). While the Lee laboratory's ideas are provocative and interesting, resolving the issues caused by heterologous antibodies as well as more precise colocalization studies are essential to understanding their results. We hope these issues can be resolved in the near future.

Given the current interest in control of centriole formation, we would love to be able to discuss the role of individual genes in the control of Naegleria basal body assembly. For example, in animal cells there have been a series of key papers dissecting the role of polo-like kinase 4 (PLK4) in the control of centriole assembly and number (e.g., $[46,47])$. In these animal cells, PLK4 localizes 
to existing centrioles and there becomes activated and appears to regulate the normal assembly of a single new centriole. In addition, overexpression of PLK4 can induce de novo centriole formation. One can imagine such roles for PLK4 in the rapid formation of basal bodies during Naegleria differentiation, but so far no Plk4 gene has been recognized in the Naegleria genome. This could be due to genetic divergence, but a comparative study indicates that orthologs of Plk4 may be limited to Ophisthokonts (animals and fungi) [48]. While Naegleria Plk1 might play the role of $P l k 4$ in the amoeboflagellate, any role of pololike kinases in this system remains a challenge for future research, particularly given the current lack of tools for gene manipulation in Naegleria cells.

\section{Strengths and future of basal body research in Naegleria}

The ease of cell culture and incredible synchrony of differentiation give Naegleria great promise as a system to understand basic mechanisms of basal body assembly. However, the lack of tools for molecular genetic analysis in Naegleria remains a very real hindrance. Despite efforts [49], there have not been any widely adopted methods of manipulating gene expression in this organism. However, the Naegleria genome encodes all the necessary molecular machinery for both meiotic recombination and RNAi, hinting that both forward and reverse genetic analysis should be feasible [11]. The recent publication of the Naegleria genome sequence [11], as well as full genome transcriptional profiling [9], opens the door to a new era of discovery and has led to a renewed interest and wider adoption of this classic model for basal body biology.

It is clear that the rapid de novo assembly of basal bodies, and the counting system that ensures that most cells assemble two basal bodies, makes Naegleria a unique system to study basal body assembly. The formation and reproduction of basal bodies in the two stable phenotypes of Tetramitus are also worthy of further study. All that is needed is that researchers meet the challenge of learning to apply molecular genetics to this fascinating system.

\section{Abbreviations \\ GPM complex: a complex containing gamma-tubulin, pericentrin, and myosin II; MKS: Meckel-Gruber syndrome; PLK: polo-like kinase; POC: proteome of centriole; SAS: spindle assembly abnormal. \\ Authors' contributions \\ LF.- $L$ and CF wrote and edited the manuscript. Both authors read and approved the final manuscript.}

\section{Authors' information}

C.F. is a Professor Emeritus at Brandeis University, and has spent a half-century striving to understand how Naegleria can undergo such an incredible metamorphosis from amoeba to flagellate in so little time. His lab has uncovered most of the basic Naegleria biology we now take for granted, and has been instrumental in developing essential tools, including growth media and methods for synchronization of cultures. He used these tools to first describe de novo basal body assembly in Naegleria, as well as to develop the multitubulin hypothesis.

As a graduate student, L.F.-L. led the Naegleria genome project and conducted a full genome transcriptional analysis of differentiation. She continues to study Naegleria basal body assembly, currently focusing on molecular tool development.

\section{Author details}

${ }^{1}$ Department of Cellular and Molecular Pharmacology, University of California, San Francisco, CA 94158, USA. ${ }^{2}$ Department of Biology, Brandeis University, Waltham, MA 02454, USA.

\section{Acknowledgements}

We especially want to thank Elaine Lai for her crucial partnership in much of the Fulton lab research and all of its writing. This work was supported by a postdoctoral fellowship from the Helen Hay Whitney Foundation awarded to L.K.F.-L.

Since this review was submitted, a paper by Fritz-Laylin et al. has been accepted (currently in press at Cytoskeleton) that shows differentiating Naegleria assemble their first centriole de novo, followed by the formation of a second centriole directly adjacent to the newly created "mother centriole."The resulting two basal bodies elongate followed quickly by flagellar outgrowth.

\section{Competing interests}

The authors declare that they have no competing interests.

Received: 7 December 2015 Accepted: 16 February 2016 Published online: 04 April 2016

\section{References}

1. De Jonckheere JF. A century of research on the amoeboflagellate genus Naegleria. Acta Protozoologica. 2002;41:309-42.

2. Fulton C. Amebo-flagellates as research partners: the laboratory biology of Naegleria and Tetramitus. In: Prescott DM, editor. Methods Cell Physiol. Vol 4. 1970. p. 341-476.

3. Fulton C. Naegleria: a research partner for cell and developmental biology. J Eukaryot Microbiol. 1993;40:520-32.

4. Fulton C. Basal bodies, but not centrioles, in Naegleria. The Journal of Cell Biology. 1971;51:826-36.

5. Fulton C, Simpson PA. Selective synthesis and utilization of flagellar tubulin: the multi-tubulin hypothesis. In: Goldman R, Pollard T, Rosenbaum J, editors. Cell Motility. 1976. p. 987-1005.

6. Lai EY, Walsh C, Wardell D, Fulton C. Programmed appearance of translatable flagellar tubulin mRNA during cell differentiation in Naegleria. Cell. 1979;17:867-78.

7. Levy YY, Lai EY, Remillard SP, Fulton C. Centrin is synthesized and assembled into basal bodies during Naegleria differentiation. Cell Motil Cytoskeleton. 1998;40:249-60.

8. Fritz-Laylin LK, Assaf ZJ, Chen S, Cande WZ. Naegleria gruberi de novo basal body assembly occurs via stepwise incorporation of conserved proteins. Eukaryot Cell. 2010;9:860-5.

9. Fritz-Laylin LK, Cande WZ. Ancestral centriole and flagella proteins identified by analysis of Naegleria differentiation. J Cell Sci. 2010;123:4024-31.

10. Fulton C, Walsh C. Cell differentiation and flagellar elongation in Naegleria gruberi. Dependence on transcription and translation. J Cell Biol. 1980;85:346-60.

11. Fritz-Laylin LK, Prochnik SE, Ginger ML, Dacks JB, Carpenter ML, Field MC, Kuo A, Paredez A, Chapman J, Pham J, Shu S, Neupane R, Cipriano M, Mancuso J, Tu H, Salamov A, Lindquist E, Shapiro H, Lucas S, Grigoriev IV, Cande WZ, Fulton C, Rokhsar DS, Dawson SC. The genome of Naegleria gruberi illuminates early eukaryotic versatility. Cell. 2010;140:631-42. 
12. Rodríguez-Ezpeleta N, Brinkmann H, Burger G, Roger AJ, Gray MW, Philippe $\mathrm{H}$, Lang BF. Toward resolving the eukaryotic tree: the phylogenetic positions of jakobids and cercozoans. Curr Biol. 2007;17:1420-5.

13. Koonin EV. The origin and early evolution of eukaryotes in the light of phylogenomics. Genome Biol. 2010;11:209.

14. Iyer LM, Anantharaman V, Wolf MY, Aravind L. Comparative genomics of transcription factors and chromatin proteins in parasitic protists and other eukaryotes. Int J Parasitol. 2008;38:1-31.

15. De Jonckheere JF. Molecular definition and the ubiquity of species in the genus Naegleria. Protist. 2004;155:89-103.

16. González-Robles A, Cristóbal-Ramos AR, González-Lázaro M, OmañaMolina M, Martínez-Palomo A. Naegleria fowleri: light and electron microscopy study of mitosis. Exp Parasitol. 2009;122:212-7.

17. Patterson M, Woodworth TW, Marciano-Cabral F, Bradley SG. Ultrastructure of Naegleria fowleri enflagellation. J Bacteriol. 1981;147:217-26.

18. Dingle AD, Fulton C. Development of the flagellar apparatus of Naegleria. J Cell Biol. 1966;31:43-54.

19. Larson DE, Dingle AD. Isolation, ultrastructure, and protein composition of the flagellar rootlet of Naegleria gruberi. J Cell Biol. 1981;89:424-32.

20. Larson DE, Dingle AD. Development of the flagellar rootlet during Naegleria flagellate differentiation. Dev Biol. 1981;86:227-35.

21. Gardiner PR, Miller RH, Marsh MC. Studies of the rhizoplast from Naegleria gruberi. J Cell Sci. 1981;47:277-93.

22. Lai EY, Remillard SP, Fulton C. The alpha-tubulin gene family expressed during cell differentiation in Naegleria gruberi. The Journal of Cell Biology. 1988;106:2035-46.

23. Schuster FL. Ultrastructure of mitosis in the amoeboflagellate Naegleria gruberi. Tissue Cell. 1975;7:1-11.

24. Walsh CJ. The structure of the mitotic spindle and nucleolus during mitosis in the amebo-flagellate Naegleria. PLoS One. 2012;7:e34763.

25. Fulton C. Cell differentiation in Naegleria gruberi. Annu Rev Microbiol. 1977;31:597-629.

26. Fritz-Laylin LK, Ginger ML, Walsh C, Dawson SC, Fulton C. The Naegleria genome: a free-living microbial eukaryote lends unique insights into core eukaryotic cell biology. Res Microbiol. 2011;162:607-18.

27. Walsh C. Synthesis and assembly of the cytoskeleton of Naegleria gruberi flagellates. J Cell Biol. 1984;98:449-56.

28. Walsh CJ. The role of actin, actomyosin and microtubules in defining cell shape during the differentiation of Naegleria amebae into flagellates. Eur J Cell Biol. 2007;86:85-98.

29. Bunting M. A Preliminary Note on Tetramitus, a Stage in the Life Cycle of a Coprozoic Amoeba. Proc Natl Acad Sci USA. 1922:8:294-300.

30. Fulton C. Transformation of Tetramitus amebae into flagellates. Science. 1970;167:1269-70.

31. Outka DE, Kluss BC. The ameba-to-flagellate transformation in Tetramitus rostratus II. Microtubular morphogenesis. J Cell Biol. 1967;35:323-46.

32. Balamuth W, Bradbury PC, Schuster FL. Ultrastructure of the amoeboflagellate Tetramitus rostratus. J Protozool. 1983;30:445-55.

33. Levy YY, Lai EY, Remillard SP, Heintzelman MB, Fulton C. Centrin is a conserved protein that forms diverse associations with centrioles and
MTOCs in Naegleria and other organisms. Cell Motil Cytoskeleton. 1996;33:298-323.

34. Woodland HR, Fry AM. Pix proteins and the evolution of centrioles. PLoS One. 2008:3:e3778.

35. Kowit JD, Fulton C. Programmed synthesis of tubulin for the flagella that develop during cell differentiation in Naegleria gruberi. Proc Natl Acad Sci USA. 1974;71:2877-81.

36. Lai EY, Remillard SP, Fulton C. A beta-tubulin gene of Naegleria encodes a carboxy-terminal tyrosine. Aromatic amino acids are conserved at carboxy termini. J Mol Biol. 1994;235:377-88.

37. Chung S, Cho J, Cheon H, Paik S, Lee J. Cloning and characterization of a divergent alpha-tubulin that is expressed specifically in dividing amebae of Naegleria gruberi. Gene. 2002;293:77-86.

38. Fulton C. Centrioles. In: Reinert J, Ursprung H, editors. Origin and continuity of cell organelles; results and problems in cell differentiation. Vol 2. New York: Springer-Verlag, Inc; 1971:170-221.

39. van Breugel $M$, Hirono $M$, Andreeva A, Yanagisawa H-A, Yamaguchi $S$, Nakazawa Y, Morgner N, Petrovich M, Ebong I-O, Robinson CV, Johnson CM, Veprintsev D, Zuber B. Structures of SAS-6 suggest its organization in centrioles. Science. 2011:331:1196-9.

40. Cottee MA, Raff JW, Lea SM, Roque H. SAS-6 oligomerization: the key to the centriole? Nat Chem Biol. 2011;7:650-3.

41. Dingle AD. Control of flagellum number in Naegleria. Temperature shock induction of multiflagellate cells. J Cell Sci. 1970;7:463-81.

42. Dingle AD. Cellular and environmental variables determining numbers of flagella in temperature-shocked Naegleria. J Protozool. 1979;26:604-12.

43. Kim H-K, Kang J-G, Yumura S, Walsh CJ, Cho JW, Lee J. De novo formation of basal bodies in Naegleria gruberi: regulation by phosphorylation. J Cell Biol. 2005:169:719-24.

44. Lee J, Kang S, Choi YS, Kim H-K, Yeo C-Y, Lee Y, Roth J, Lee J. Identification of a cell cycle-dependent duplicating complex that assembles basal bodies de novo in Naegleria. Protist. 2015;166:1-13.

45. Suh MR, Han JW, No YR, Lee J. Transient concentration of a gammatubulin-related protein with a pericentrin-related protein in the formation of basal bodies and flagella during the differentiation of Naegleria gruberi. Cell Motil Cytoskeleton. 2002;52:66-81.

46. Ohta M, Ashikawa T, Nozaki Y, Kozuka-Hata H, Goto H, Inagaki M, Oyama M, Kitagawa D. Direct interaction of Plk4 with STIL ensures formation of a single procentriole per parental centriole. Nat Commun. 2014;5:5267.

47. Lopes CAM, Jana SC, Cunha-Ferreira I, Zitouni S, Bento I, Duarte P, Gilberto S, Freixo F, Guerrero A, Francia M, Lince-Faria M, Carneiro J, BettencourtDias M. PLK4 trans-autoactivation controls centriole biogenesis in space. Dev Cell. 2015;35:222-35.

48. Carvalho-Santos Z, Machado P, Branco P, Tavares-Cadete F, RodriguesMartins A, Pereira-Leal JB, Bettencourt-Dias M. Stepwise evolution of the centriole-assembly pathway. J Cell Sci. 2010;123:1414-26.

49. Song K-J, Jeong S-R, Park S, Kim K, Kwon M-H, Im K-I, Pak JH, Shin $\mathrm{H}$-J. Naegleria fowleri: functional expression of the $\mathrm{Nfa} 1$ protein in transfected Naegleria gruberi by promoter modification. Exp Parasitol. 2006;112:115-20.

\section{Submit your next manuscript to BioMed Central and we will help you at every step:}

- We accept pre-submission inquiries

- Our selector tool helps you to find the most relevant journal

- We provide round the clock customer support

- Convenient online submission

- Thorough peer review

- Inclusion in PubMed and all major indexing services

- Maximum visibility for your research

Submit your manuscript at www.biomedcentral.com/submit
() Biomed Central 